$$
\begin{aligned}
& \text { اهميت آرايه شناسى صفات ريختى در Fل كندم (تيره كاسنيان) } \\
& \text { سحرناز راكىزاده'، فريده عطار 'و آرش ستوده׳ } \\
& \text { 'هرباريوم مر كزى دانشكده علوم، دانشگاه تهران، ايران؛ 'ديارتمان اكولوزى و يراكندگى زيستى، دانشگاه تولوز، تولوز، فرانسه } \\
& \text { arash.sotoodeh@ac-toulouse.fr مسئول مكاتبات: آرش ستوده، }
\end{aligned}
$$

جكيده. ريختشناسى فندقه در Centaurea در وא آرايه مطالعه شده است. در اين بررسى براى جداسازى بخشها، 19 صفت تشخيصى ارائه شده است. بر اساس

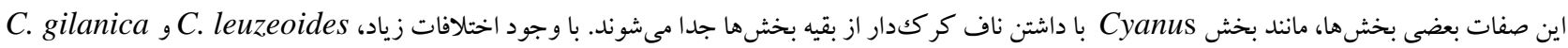

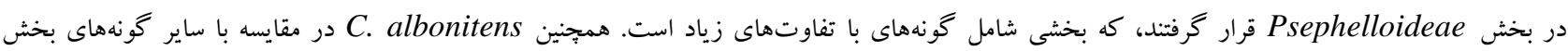
Phaeopappus

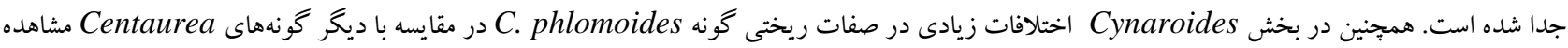

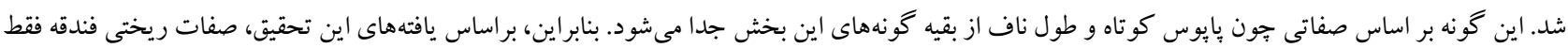

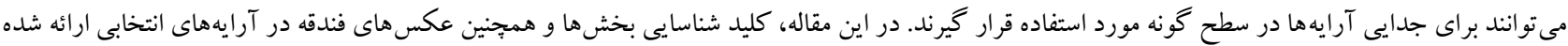

$$
\text { وازههاى كليدى. ايران، بايوس، فلورا ايرانيكا، كليدشناسايى، مر كبان }
$$

\title{
Taxonomic significance of achene morphology in the genus Centaurea $\mathrm{L}$. (Asteraceae)
}

\author{
Saharnaz Rakizadeh ${ }^{1}$, Farideh Attar $^{1} \&$ Arash Sotoodeh ${ }^{2}$ \\ ${ }^{1}$ School of Biology, College of science, University of Tehran, Tehran, Iran; ${ }^{2}$ Université de Toulouse III, EDB, \\ UMR5174, Toulouse, France. \\ Correspondent author: Arash Sotoodeh, arash.sotoodeh@ac-toulouse.fr
}

\begin{abstract}
Achene morphology of 49 taxa of the genus Centaurea L. was studied in terms of 19 different characteristics. On the basis of the variation in these features, some sections, such as sect. Cyanus with hairy hilum, were separated. Despite various differences, $C$. leuzeoides and $C$. gilanica were categorized in the section Psephelloideae, a section with lots of character variations within its species. Also, $C$. albonitens has different characteristics in comparison with other taxa within section Phaeopappus, for example features such as pappus color and the ratio of pappus length to that of achene separated the species from other members of the section. The last but not the least, highly different characteristics were observed in the achene morphology of $C$. phlomoides as compared with other species in section Cynaroides. Differences such as short pappus and hilum length were found to separate C. phlomoides from other members of sect. Cynaroides. In conclusion, on the basis of the findings of this research, most achene morphological characteristics can be appropriately used as key features for the differentiation of sections in the genus Centaurea. An identification key based on the features of the achene, images related to the achene of the studied species were also presented.
\end{abstract}

Keywords. Compositae, Flora Iranica, identification key, Iran, pappus 


\section{INTRODUCTION}

Centaurea L. (Asteraceae), with 350 to 600 species of herbaceous thistle-like plants in the world (Heywood, 1979; Hickey \& King, 1981), is found only in the North of the equator, mostly in the Eastern hemisphere. The Middle-East and its surrounding regions are particularly species-rich with regard to the genus Centaurea (Davis et al., 1988; Wagenitz, 1980). This genus belongs to subtribe Centaurinae, Tribe Cardueae, subfamily Carduoideae and family Asteraceae (Judd et al., 2008; Bremer et al., 2009). Based on Flora Iranica (Wagenitz, 1980), Centaurea, with 88 species in the Iranian plateau, is divided into 28 sections. The infrageneric classifications of the genus Centaurea were mainly based on features such as plant duration, characteristics of involucres (shape of phyllaries, cilias, terminal spine tipped or non spine tipped of bracts), and color of florets and position of leaves. Moreover, flower colors and heights were the other criteria considered to be taxonomically important. Centaurea is one of the largest genera in Iran with almost 89 species, of which 76 species are endemic (Wagenitz, 1980; Ghahreman \& Attar, 1999; Shabestari et al., 2013a, 2013b; Negaresh et $a l ., 2014)$. This genus is a bushy plant with ciliate (C. depressa M.Bieb.) or spiny (C. kandavanensis Wagenitz) phyllaries with white, yellow, pink or purple flowers. Some phyllaries overlapped in several rows, with tips variously spiny or mucronate and margins pinnate or entire. The stems of the plants are long and erect (C. cheiranthifolia Willd.), rarely acaulescent $(C$. rhizantha Tchich.), short $(C$. aucheri (DC.) Wagenitz) or prostrate ( $C$. incanescens (DC.) Sch.Bip.). Leaves are entire, pinnatifid, pinnatisect, with different shapes, and are covered with gray hairs (gray-tomentose), rarely dense arachnoid (C. luristanica Rech.f.) or glabrous (C. koeieana Bornm.). Achenes are oblong, rarely triangular, 2.5-3.5 mm long, with apex flattened, tapered to a rounded, having a laterally notched base. Pappus are often white (colorful pappus is an exception, as seen in some species like C. aucheri), composed of unequal, stiff, minutely barbellate or tiny, flat scales (Fig. 1) (Wagenitz, 1980). As it was mentioned before, the achene characteristics have not been studied thoroughly, or rarely considered to be taxonomically important (Maleev, 1971; Boissier, 1875; Wagenitz, 1975, 1980; Shabestari et al., 2013a, 2013b; Bona, 2014; Negaresh et al., 2014; Ranjbar \& Negaresh, 2014). In this paper, achene variation in 49 taxa (44 species and 5 subspecies) belonging to 24 sections was investigated. The criteria were highly focused on the achene and pappus characteristics, and the study has been concluded with achene-based identification key for the sections of the genus Centaurea.

\section{MATERIALS \& METHODS}

Achenes of 44 species and five subspecies of 24 sections belonging to the genus Centaurea were collected from specimens preserved in the Central Herbarium of Tehran University (TUH), as shown in Table 1. The sections Phalolepis (Cass.) DC., Grossheimia (Sosn. \& Takht.) Dittrich and Czerniakovskya (Czerep.) Wagenitz have been excluded from the study, since no proper specimens of these sections were available.

Then, achenes were studied and photos were taken by means of a Dinolite hand-held digital microscope with a magnification of $180 x$.

In order to investigate the characteristics of achene in the specimens studied, 19 criteria were sorted out. The selected characteristics included features such as appendage length and color, hilum length, achene length, pappus-achene connection length, color and shape of achene, pappus and inner pappus type, and ratio of the length of pappus to that of achene. The complete list of the characteristics studied is presented in Table 2.

\section{RESULTS}

As it is shown in Tables 2 and 3,19 criteria were taken into consideration in characterizing and sorting out the differences among the 49 taxa studied (44 species and 5 subspecies). Some of these characteristics and their importance are explained below.

- Appendage of hilum: the first and the most important characteristic key which separates the taxa studied into two main parts, based on whether they have appendiculate hilum or exappendiculate hilum.

- Color of appendage: section Stizolophus (Cass.) DC. (C. balsamita Lam.) becomes distinct by redbrown appendage among all other sections.

- Achene's shape: it varies among the taxa studied; some are oblong ( $C$. behen Lam.), fusiform ( $C$. amadanensis Sch.Bip.) or urceolate (C. kotschyi (Boiss.) Hayek), while others are triangular ( $C$. glastifolia L.), elliptic (C. geluensis Boiss. \& Hausskn. ex Boiss.) or rectangular (C. gaubae (Bornm.) Wagenitz).

- Achene's size: most of the taxa studied have normal size (2.5-3.5 mm long), while some can be seen in inflated form, for example $C$. zuvandica (Sosn.) Sosn. in sect. Psephellus (Cass.) DC. and $C$. incanescens (Fisch. \& C.A.Mey. ex DC.) Sosn. in sect. Amblyopogon Fisch. \& C.A.Mey. ex DC. 

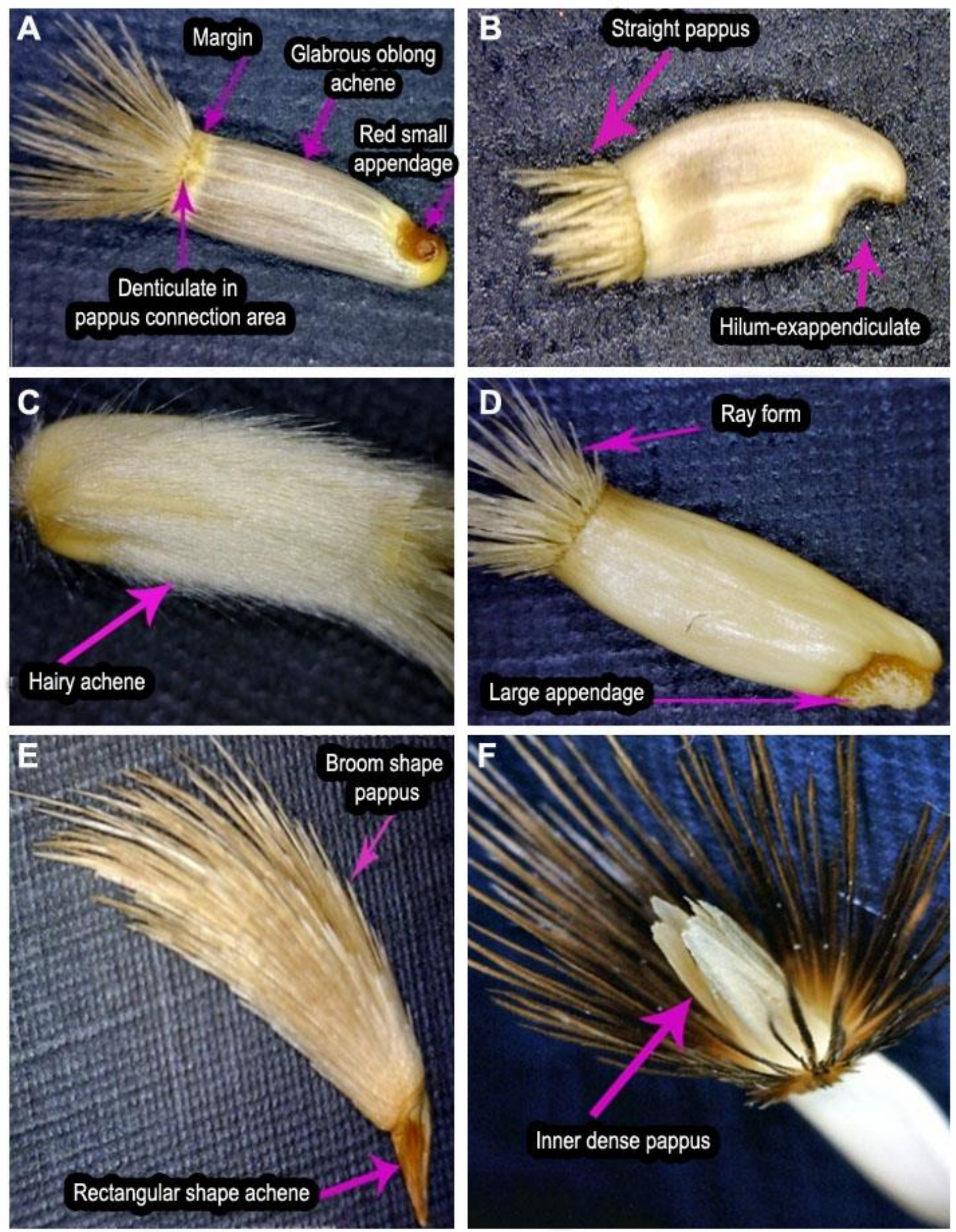

Fig 1. Different types of achene and pappus. A. Centaurea balsamita subsp. kermanensis (Bornm.) Wagenitz. B. C. iberica Trevir. ex Spreng. C. C. luristanica Rech.f. D. C. ustulata DC. E. C. albonitens Turrill, F. C. leuzeoides Walp.

- Pappus length: pappus are shorter than the achene in the first group (C. phlomoides Boiss. \& Hausskn. ex Boiss.), while in the second group the pappus are longer than the achene ( $C$. luristanica), and in the third group the lengths of achene and pappus are equal (C. bruguierana Hand.-Mazz.). Differences in pappus length among different sections as well as within certain sections is also observed (C. iberica and 
Table 1. Voucher specimens used in achene characterization.

\begin{tabular}{|c|c|c|c|}
\hline Species & Locality & $\begin{array}{c}\text { Height } \\
\text { (m) }\end{array}$ & $\begin{array}{c}\text { Collector \& Herbarium } \\
\text { nummber }\end{array}$ \\
\hline Centaurea lachnopus Rech.f. & Semnan: ca 15 from Semnan to Firouz kuh & 1130 & $\begin{array}{c}\text { Ghahreman, Mozaffarian } 5822 \\
\text { (TUH) }\end{array}$ \\
\hline $\begin{array}{l}\text { C. balsamita subsp. balsamita } \\
\text { Lam. }\end{array}$ & Golestan: Golestan National Park & s.n. & $\begin{array}{l}\text { Ghahreman, Mozaffarian, Attar } \\
25438 \text { (TUH) }\end{array}$ \\
\hline $\begin{array}{l}\text { C. balsamia subsp. balsamita } \\
\text { Lam. }\end{array}$ & $\begin{array}{l}\text { Azarbayejan: Marand to Evoghli, Kushksary } \\
\text { to Erelan }\end{array}$ & 1360 & $\begin{array}{c}\text { Ghahreman, Mozaffarian } 9747 \\
\text { (TUH) }\end{array}$ \\
\hline $\begin{array}{l}\text { C. balamita subsp. balsamia } \\
\text { Lam. }\end{array}$ & Azarbayejan; Urmieh, Balanesh & 1350 & Ghahreman, Attar 21319 (TUH) \\
\hline $\begin{array}{l}\text { C. balsamita subsp. balsamita } \\
\text { Lam. }\end{array}$ & Kermanshah: $40 \mathrm{~km}$, to Paveh & 1550 & $\begin{array}{c}\text { Attar, Dadjou, Mehdigholi, } \\
\text { Okhovat } 14237 \text { (TUH) }\end{array}$ \\
\hline $\begin{array}{l}\text { C. balsamita subsp. kermanensis } \\
\text { (Bornm.) Wagenitz }\end{array}$ & $\begin{array}{c}\text { Khorasan: Neishabour road, Mt. Binaloud } \\
\text { Kouh }\end{array}$ & 1250 & Ghahreman, Attar 21916 (TUH) \\
\hline $\begin{array}{l}\text { C. balsamita subsp. kermanensis } \\
\text { (Bornm.) Wagenitz }\end{array}$ & Fars: Shiraz, Psargad & 1845 & Ghahreman, Attar 22515 (TUH) \\
\hline C. aggregata Fisch. \& C.A.Mey. & Kermanshah: $5 \mathrm{~km}$ after Paveh to Nasoud & 1550 & $\begin{array}{l}\text { Ghahreman, Attar, Mehdigholi } \\
22376 \text { (TUH) }\end{array}$ \\
\hline C. aggregata Fisch. \& C.A.Mey. & Kermanshah: 50 km Paveh & 1550 & Ghahreman, Attar 21202 (TUH) \\
\hline C. aggregata Fisch. \& C.A.Mey. & $\begin{array}{c}\text { Kordestan: Ghorv: to Sanandaj: } 45 \mathrm{~km} \text { to } \\
\text { Sanandaj }\end{array}$ & 1500 & Ghahreman, Attar 19647 (TUH) \\
\hline C. aggregata Fisch. \& C.A.Mey. & $\begin{array}{l}\text { Kordestan: Marivan to Sanandaj from old } \\
\text { road Gardane Geran }\end{array}$ & 1320 & $\begin{array}{l}\text { Ghahreman, Mozaffarian } 18303 \\
\text { (TUH) }\end{array}$ \\
\hline C. ovina Pall. ex Willd. & Azarbayejan: Ardebil, $10 \mathrm{~km}$ to Ahar & 1370 & Attar, Dadjou 17237 (TUH) \\
\hline C. ovina Pall. ex Willd. & $\begin{array}{c}\text { Azarbayejan: Ardebil to Germi, } 10 \mathrm{~km} \text { to } \\
\text { Germi }\end{array}$ & 1860 & Attar, Dadjou 14679 (TUH) \\
\hline C. ammocyanus Boiss. & Alborz: Near Hashtgerd, 30 km WD Karaj & 1250 & Esfandiari 11445-TUH \\
\hline C. pulchella Ledeb. & Azarbayejan: Tabriz on the road to Lighvan & 2280 & $\begin{array}{l}\text { Ghahreman, Mozaffarian } 17376 \\
\text { (TUH) }\end{array}$ \\
\hline C. pulchella Ledeb. & Khorasan: mt. Kashmar & 1060 & Ghahreman, Attar 27324 (TUH) \\
\hline C. hyrcanica Bornm. & Mazandaran: Nowshahr, Kheiroud forest & 20 & Syadati, Moradi 40196 (TUH) \\
\hline C. hyrcanica Bornm. & Gilan: Ispili & 1580 & Syadati $18489(\mathrm{TUH})$ \\
\hline C. kotschyi (Boiss.) Hayek & Mazandara: road of Karaj - Chalous & 40 & Nazaryan $33460($ TUH $)$ \\
\hline C. kotschyi (Boiss.) Hayek & Tehran: Chalous road, slope of Kandavan & s.n. & $\begin{array}{c}\text { Ghahreman, Mozaffarian } 9780 \\
\text { (TUH) }\end{array}$ \\
\hline C. ispahanica Boiss. & Isfahan: Shahreza, Samirom, Kouhravi, & 2450 & $\begin{array}{c}\text { Ghahreman, Mozaffarian } 18233 \\
\text { (TUH) }\end{array}$ \\
\hline C. rhizantha Tchich. & $\begin{array}{c}\text { Azarbayejan: Arasbaran, protected Area, } \\
\text { Kouhe Kalan }\end{array}$ & 1105 & Attar, Dadjou 17671 (TUH) \\
\hline C. rhizantha Tchich. & $\begin{array}{c}\text { Azarbayejan: Marand, Zunuz, between } \\
\text { Zunuzagh and Kuhkamar }\end{array}$ & 1700 & $\begin{array}{c}\text { Ghahreman, Mozaffarian } 17405 \\
\text { (TUH) }\end{array}$ \\
\hline C. glastifolia $\mathrm{L}$. & Azarbayejan: Marand road of Zunuz & 1700 & Ghahreman, Attar 21296 (TUH) \\
\hline C. glastifolia $\mathrm{L}$. & $\begin{array}{l}\text { Azarbayejan: Khoy road of Ghotur, Razi, } 2 \\
\text { km of bus station }\end{array}$ & 1160 & Ghahreman, Attar 21992 (TUH) \\
\hline $\begin{array}{l}\text { C. aucheri subsp. aucheri (DC.) } \\
\text { Wagenitz }\end{array}$ & $\begin{array}{c}\text { Kordestan: Sanandaj, } 28 \text { km to Divan } \\
\text { Darreh }\end{array}$ & 1840 & $\begin{array}{l}\text { Attar, Dadjou, Mehdigholi, } \\
\text { Okhovat } 14293 \text { (TUH) }\end{array}$ \\
\hline $\begin{array}{l}\text { C. aucheri subsp. aucheri (DC.) } \\
\text { Wagenitz }\end{array}$ & $\begin{array}{l}\text { Hamadan: Kubardar Ahang to Ghohord, } \\
\text { Keitou, Kouhe Keiton }\end{array}$ & 1800 & Mozaffarian 64603 (TUH) \\
\hline $\begin{array}{l}\text { C. aucheri subsp. aucheri (DC.) } \\
\text { Wagenitz }\end{array}$ & $\begin{array}{l}\text { Azarbayejan sharqi: N slopes of Mishov- } \\
\text { Dagh, south of the road }\end{array}$ & 1400 & Podlesh, Zarre 55267 (TUH) \\
\hline $\begin{array}{l}\text { C. aucheri subsp. szowittsii } \\
\text { (Boiss.) Wagenitz }\end{array}$ & Azarbayejan: Gardane Yam, Mishodagh & 1400 & $\begin{array}{c}\text { Ghahreman, Aghostin, } \\
\text { Sheikholeslami } 11444 \text { (TUH) }\end{array}$ \\
\hline $\begin{array}{l}\text { C. aucheri subsp. szowittsii } \\
\text { (Boiss.) Wagenitz }\end{array}$ & $\begin{array}{c}\text { Markazi: } 70 \mathrm{~km} \mathrm{NW} \text { of Saveh village of } \\
\text { Bandamir }\end{array}$ & 1920 & Ghaffari, 4681 (TUH) \\
\hline C. aucheri-elbursensis Wagenitz & $\begin{array}{c}\text { Azarbayejan: Myaneh, Bostanabad, } 35 \mathrm{~km} \\
\text { Bostanabad }\end{array}$ & 1740 & Ghaffari, 11627 (TUH) \\
\hline C. albonitens Turrill & Azarbayejan: Sirvan, from Yam to Tabriz & 1900 & $\begin{array}{c}\text { Ghahreman, Aghostin, } \\
\text { Sheikholeslami } 11477 \text { (TUH) }\end{array}$ \\
\hline C.albonitens Turrill & Azarbayejan: Tabriz, $20 \mathrm{~km}$ to Marand & 1360 & Ghaffari, 6642 (TUH) \\
\hline $\begin{array}{l}\text { C.geluensis Boiss. \& Hausskn. } \\
\text { ex Boiss. }\end{array}$ & Lorestan: Khorramabad, Sefidkou & 1720 & Veis Karami 23715 (TUH) \\
\hline C. gigantea Sch.Bip. ex Boiss. & Lorestan: Khoramabad, road of Sefid Dasht & 1142 & $\begin{array}{c}\text { Ghahreman, Attar, Dadjou } 21840 \\
\text { (TUH) }\end{array}$ \\
\hline
\end{tabular}


Table 1. continue ...

\begin{tabular}{|c|c|c|c|}
\hline Species & Locality & $\begin{array}{l}\text { Height } \\
(\mathbf{m})\end{array}$ & $\begin{array}{c}\text { Collector \& Herbarium } \\
\text { nummber }\end{array}$ \\
\hline $\begin{array}{l}\text { C. imperialis Hausskn. ex } \\
\text { Bornm. }\end{array}$ & $\begin{array}{c}\text { Kordestan: Marivan to Baneh } 50 \mathrm{~km} \text { to } \\
\text { Baneh }\end{array}$ & 1540 & Ghahreman, Attar 19667 (TUH) \\
\hline $\begin{array}{l}\text { C. phlomoides Boiss. \& } \\
\text { Hausskn. ex Boiss. }\end{array}$ & Kermanshah: Paveh & 1550 & Attar, Mirtadzadini 19857 (TUH) \\
\hline $\begin{array}{l}\text { C. amadanensis subsp. } \\
\text { gymnoclada (Jaub. \& Spach) } \\
\text { Negaresh }\end{array}$ & Kordestan, Marivan, Ghamishlu & 1320 & Ghahreman, Attar 19650 (TUH) \\
\hline $\begin{array}{l}\text { C. amadanensis subsp. } \\
\text { amadanensis } \text { Sch.Bip. }\end{array}$ & $\begin{array}{c}\text { Lorestan: Khoramabad, } 50 \mathrm{~km} \text { after } \\
\text { bifurcation of Khorramabad- Sefid Dasht }\end{array}$ & $1142 \mathrm{~m}$ & $\begin{array}{c}\text { Ghahreman, Attar, Ghaffari } 21839 \\
\text { (TUH) }\end{array}$ \\
\hline C. nemecii Nábělek & Kordestan: Sanandaj, Salavat Abad pass & $1700 \mathrm{~m}$ & $\begin{array}{l}\text { Ghahreman, Mozaffarian } 18295 \\
\text { (TUH) }\end{array}$ \\
\hline C. koeieana Bornm. & Lorestan: Khorramabad, Delbar & $1100 \mathrm{~m}$ & Veis Karami $23712($ TUH) \\
\hline C. pabotii Wagenitz & $\begin{array}{c}\text { Chaharmahal-e- Bakhtiari Lordegan to } \\
\text { Dashte Armand }\end{array}$ & $1585 \mathrm{~m}$ & Mozaffarian 54658 (TUH) \\
\hline C. behen Lam. & $\begin{array}{l}\text { Lorestan: Khorramabad, Cham-Divan, Chal- } \\
\text { e-Ahmad }\end{array}$ & $1440 \mathrm{~m}$ & Veis Karami 23713 (TUH) \\
\hline C. solstitialis Asso & Azarbayejan: between Ardebil-Kivy & $1480 \mathrm{~m}$ & Sheikholeslami $11510(\mathrm{TUH})$ \\
\hline C. pseudosinaica Czerep. & $\begin{array}{l}\text { Hormozgan: Bandar Abbas, near Sarkhon, } \\
\text { Radar site }\end{array}$ & $75 \mathrm{~m}$ & $\begin{array}{c}\text { Ghahreman, Mozaffarian } 5357 \\
\text { (TUH) }\end{array}$ \\
\hline C. iberica Trevir. ex Spreng. & Gilan: Langroud. Chamkhaleh & $-26 m$ & Naghinezhad 27549 (TUH) \\
\hline C. iberica Trevir. ex Spreng. & Gilan: Lngroud, Chamkhaleh & $-26 \mathrm{~m}$ & Naghinezhad 27548 (TUH) \\
\hline C. bruguierana Hand.-Mazz. & Khuzestan: Mahshahr & & Ghahreman \& Attar23340 (TUH) \\
\hline C. bruguierana Hand.-Mazz. & $\begin{array}{c}\text { Kordestan: inter Gilan e Gharb and Ghasre } \\
\text { Shirin }\end{array}$ & $360 \mathrm{~m}$ & Ghahreman $11456(\mathrm{TUH})$ \\
\hline C. sosnowskyi Grossh. & Gilan: between Ispili and Leih & $1580 \mathrm{~m}$ & Saiydi 18490-(TUH) \\
\hline C. kandavanensis Wagenitz & Golestan: before Nardin to Tange rah & $465 \mathrm{~m}$ & Ghahreman, Attar 21930-(TUH) \\
\hline C. luristanica Rech.f. & Khuzestan: Ize, Darre Sansan & & Attar, Dadjou 17723-(TUH) \\
\hline C. leuzeoides Walp. & Gorgan: Golestan National park, Almeh & - & $\begin{array}{c}\text { Ghahreman, Mozaffarian } 5903 \\
\text { (TUH) }\end{array}$ \\
\hline C. gilanica Bornm. & $\begin{array}{l}\text { Hamedan: Famenin; Ghorveh, } \\
\text { Karafs, Mnts N.E of Karafs }\end{array}$ & $1790 \mathrm{~m}$ & Mozaffarian 64542 (TUH) \\
\hline C. zuvandica (Sosn.) Sosn. & $\begin{array}{l}\text { Mazandaran: Kandavan road of Chalous- } \\
\text { Haraz, Yoush }\end{array}$ & $2230 \mathrm{~m}$ & Ghaffari 21229 (TUH) \\
\hline C. phaeopappoides Bordz. & $\begin{array}{l}\text { Azarbayejan: Siah cheshme Baron village, } \\
\text { around Zarzor Chuch }\end{array}$ & $1750 \mathrm{~m}$ & Mozaffarian $71130(\mathrm{TUH})$ \\
\hline
\end{tabular}

C. hyalolepis Boiss. in section Calcitrapa DC.).

- Pappus color: most pappus are cream, brown or milky white, but species in section Phaeopappus (DC.) O.Hoffm. (except C. albonitens), Psephelloideae (Boiss.) Sosn. and Xanthopsis are distinguished by their purple or black pappus.

- Inner dense pappus: this characteristic can be seen in sections Hyalea, Mesocentron (C. solstitialis), Tetramorphaea, Acrocentron, Psephelloideae (C. leuzeoides), Odontolophoideae, Xanthopsis (DC.) Wagenitz \& Hellwig and Cyanus (Miller) DC. (except $C$. elbrusensis Boiss. \& Buhse).

Pappus form: pappus, either short or long, has different forms, most of which in ray form are separated from other taxa (C. aucheri), while some others are dense and straight (C. ispahanica Boiss.). Though $C$. albonitens in section Phaeopappus with broom-shaped pappus and $C$. incanescens in section Amblyopogon with short truncate and oblique pappus are very different among all species studied in other sections.

- Connection area of pappus to achene: it is mostly denticulate or rarely entire (C. gilanica Bornm.).
The section Cyanus has hairy hilum area, except for the C. elbursensis.

As it is observed in Tables 2 and 3, there are differences in achene's characteristics among sections, even in one unique $C$. lachnopus Rech.f. in sect. Centaurea with distinct white appendage coming out of hilum (Fig. 2 A).

The members of sect. Acrolophus (Cass.) DC. \& Ammocyanus Boiss. are similar in achene chracteristics, and based on Wagenitz (1980), these species have apparent similar morphology too (Fig. $2 \mathrm{D}, \mathrm{E} \& \mathrm{~F}$ ). Based on Tables 2 and 3, hilum in $C$. hyrcanica Bornm. in sect. Jacea (Miller) DC., is concave, semi-circular and no appendage can be seen in it (Fig. $2 \mathrm{H}$ ).

In sect. Rhizocalathium Tzvelev (Fig. 2 J, K \& L) appendage is seen out of hilum (similar to sect. Centaurea), and a narrow brown margin is seen in the bottom of achene in hilum opening.

Sect. Phaeopappus (C. aucheri with three subspecies) are similar, especially in having purple pappus and the length of pappus in comparison with achene length (Fig. 3 N- P), while C. albonitens in the same section 
Table 2. Characterizations used for achene differentiation (measurement in $\mathrm{mm}$ ).

Abbreviations: App=appendage, App.L=appendage length, App.C=appendage color, $\mathbf{H}=$ hilum, H.L=hilum length, A.L=achene length, A.W=achene width, A.C=achene color, A.S=achene shape, int=intangible, -=glabrous, +/=pubsent,$+=$ normal, $++=$ fairly high,$+++=$ highly.

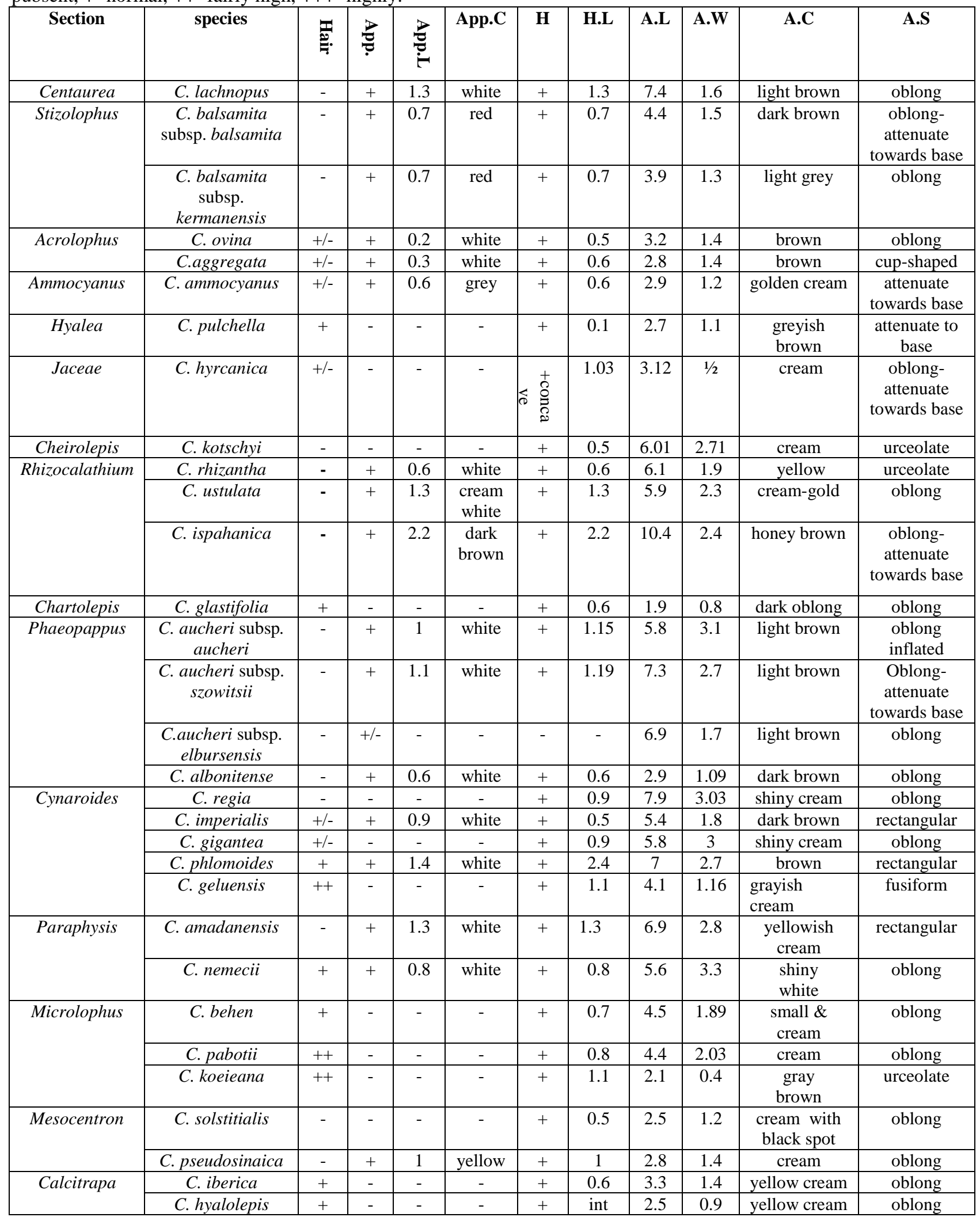


Table 2. continue ...

\begin{tabular}{|c|c|c|c|c|c|c|c|c|c|c|c|}
\hline Section & species & 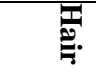 & $\frac{2}{2}$ & $\stackrel{2}{0}$ & $\underset{\text { Cpp. }}{\text { App. }}$ & $\mathbf{H}$ & H.L & $\begin{array}{l}\text { A. } \\
\text { L }\end{array}$ & $\begin{array}{l}\text { A. } \\
\text { W }\end{array}$ & A.C & A.S \\
\hline Tetramorphaea & C. bruguierana & + & - & - & - & + & 0.3 & 2 & 0.9 & yellow caramel & oblong \\
\hline \multirow[t]{3}{*}{ Acrocenteron } & C. sosnowskyi & ++ & - & - & - & + & 1.5 & 5.4 & 2.3 & amber colored & oblong \\
\hline & C. kandavanensis & +++ & - & - & - & + & 1.2 & 5.4 & 2.3 & amber colored & oblong \\
\hline & C. luristanica & +++ & - & - & - & + & 1.05 & 6.2 & 2.5 & amber colored & oblong \\
\hline \multirow[t]{2}{*}{ Psephelloideae } & C. leuzeoides & - & - & - & - & + & 1.07 & 8.1 & 2.9 & shiny white & $\begin{array}{l}\text { fatty } \\
\text { oblong }\end{array}$ \\
\hline & C. gilanica & $+/-$ & + & 1.01 & white & + & 1.01 & 6.8 & 2.7 & yellow honey & oblong \\
\hline Psephellus & C. zuvandica & + & + & 1.4 & white & + & 1.4 & 5.3 & 2.6 & yellow cream & urceolate \\
\hline Amblyopogon & C. incanescens & - & - & - & - & + & 2.5 & 7.3 & 4.17 & cream white & urceolate \\
\hline Odontolophoideae & C.phaeopappoides & $\begin{array}{c}++ \\
\text { pilose }\end{array}$ & + & 0.7 & white & + & 0.8 & 3.8 & 1.6 & light brown & elliptic \\
\hline Uralepis & C. gaubea & $\begin{array}{c}+ \\
\text { pilose }\end{array}$ & + & 1.2 & white & + & 1.2 & 2.8 & 1.3 & yellow honey & rectangular \\
\hline Xanthopsis & C. xanthocephala & - & + & 1.2 & white & + & 1.3 & 6.8 & 2.5 & amber colored & elliptic \\
\hline Cyanus & C. cyanus & + & + & 2 & white & + & 2.3 & 4.6 & 2.4 & yellow brown & triangular \\
\hline \multirow[t]{4}{*}{ Cyanus } & C. cheiranthifolia & - & + & 1.1 & white & + & 1.5 & 4.4 & 2.08 & yellow cream & oblong \\
\hline & C. depressa & + & + & 2.9 & white & + & 2 & 4.9 & 2.6 & light brown & oblong \\
\hline & C. elbursensis & + & + & 1.9 & white & + & 2.1 & 5.6 & 1.7 & brown & oblong \\
\hline & C. triumfetti & + & + & 1.4 & white & + & 1.5 & 4.8 & 1.9 & black yellow & oblong \\
\hline
\end{tabular}
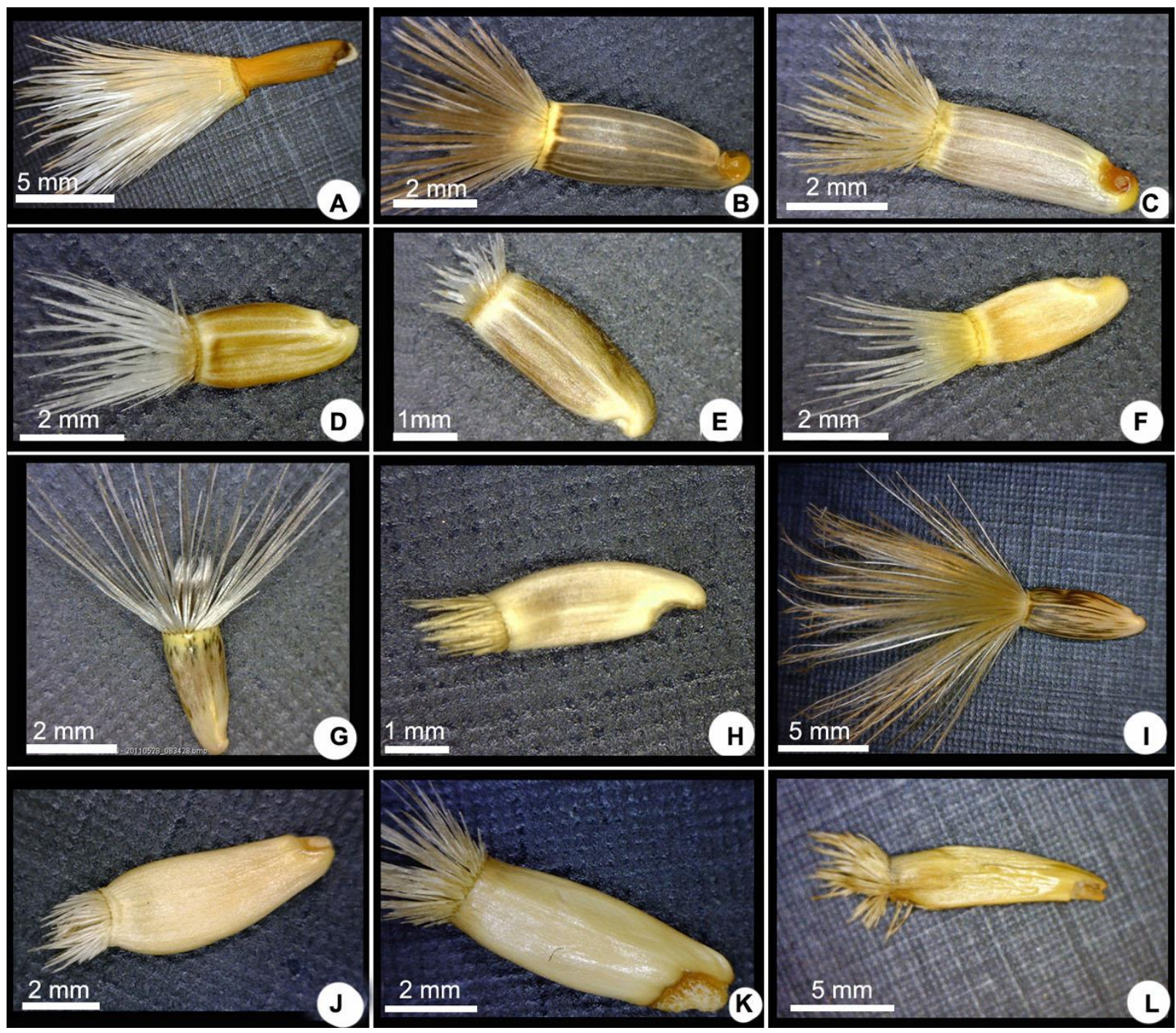

Fig. 2. A. Centaurea. lachnopus. B. C. balsamita subsp. balsamita. C. C. balsamita subsp. kermanensis. D. C. aggregate. E. C. ovina. F. C. ammocyanus. G. C. pulchella. H. C.hyrcanica. I. C. kotschyi. J. C. rhizantha. K. C. ustulata. L. C. ispahanica. 
is observed to have white cream pappus (Fig. 3 Q). Moreover, outer pappus are shorter in comparison with the inner part.

C. nemecii Nábělek in sect. Paraphysis (DC.) Wagenitz has bright milky achene, and similar color in short outer pappus and long inner pappus (Fig. 3 X). Sect. Microlophus (Cass.) DC. is noticeable in terms of the angled form of its hilum (Fig. 4 A \& B). Achene of C. koeieana in sect. Microlophus is covered with dense hairs (Fig. 4 C). C. iberica in sect. Calcitrapa is distinguished by short and scarce hairs on its hilum (Fig. 4 F).

Species belong to sect. Acrocentron (Cass.) DC. are fully covered with soft hairs and have inner dense pappus (I.D.P) which is observable in all of the three species of the section, especially in $C$. sosnowskyi Grossh. which has scaly inner dense pappus (Fig. 4 I ). C. zuvandica (Fig. 5 N) in sect. Psephellus is similar to members of sects. Centaurea and Rhizocalathium in that it has clear appendage, out of hilum, and similar to the members of section Microlophus in that it has truncate hilum.

C. leuzeoides (Fig. $4 \mathrm{~L}$ ) in sect. Psephelloideae has black pappus, shiny white achene and inner dense pappus.

The achene in $C$. incanescens is large and inflated with a concave semi-circular hilum, short, dense, truncate and oblique pappus (Fig. 5 O). $C$. phaeopappoides (Fig. $5 \mathrm{P}$ ) in sect. Odontolophoideae is similar to the members of the sect. Centaurea, Rhizocalathium and Psephellus as its appendage is out of hilum.

Three sects. Phaeopappus, psephelloideae (C. gilanica, Fig. $5 \mathrm{M}$ ) and Xanthopsis have purple pappus. As it was mentioned above, species in sect. Cyanus, especially $C$. cheiranthifolia, $C$. triumphetti, and $C$. depressa have hairs on their hilum (Fig. $5 \mathrm{~S}, \mathrm{U}$ and $\mathrm{V}$ ), while the presence of hairs on the hilum is scarce in other sections.

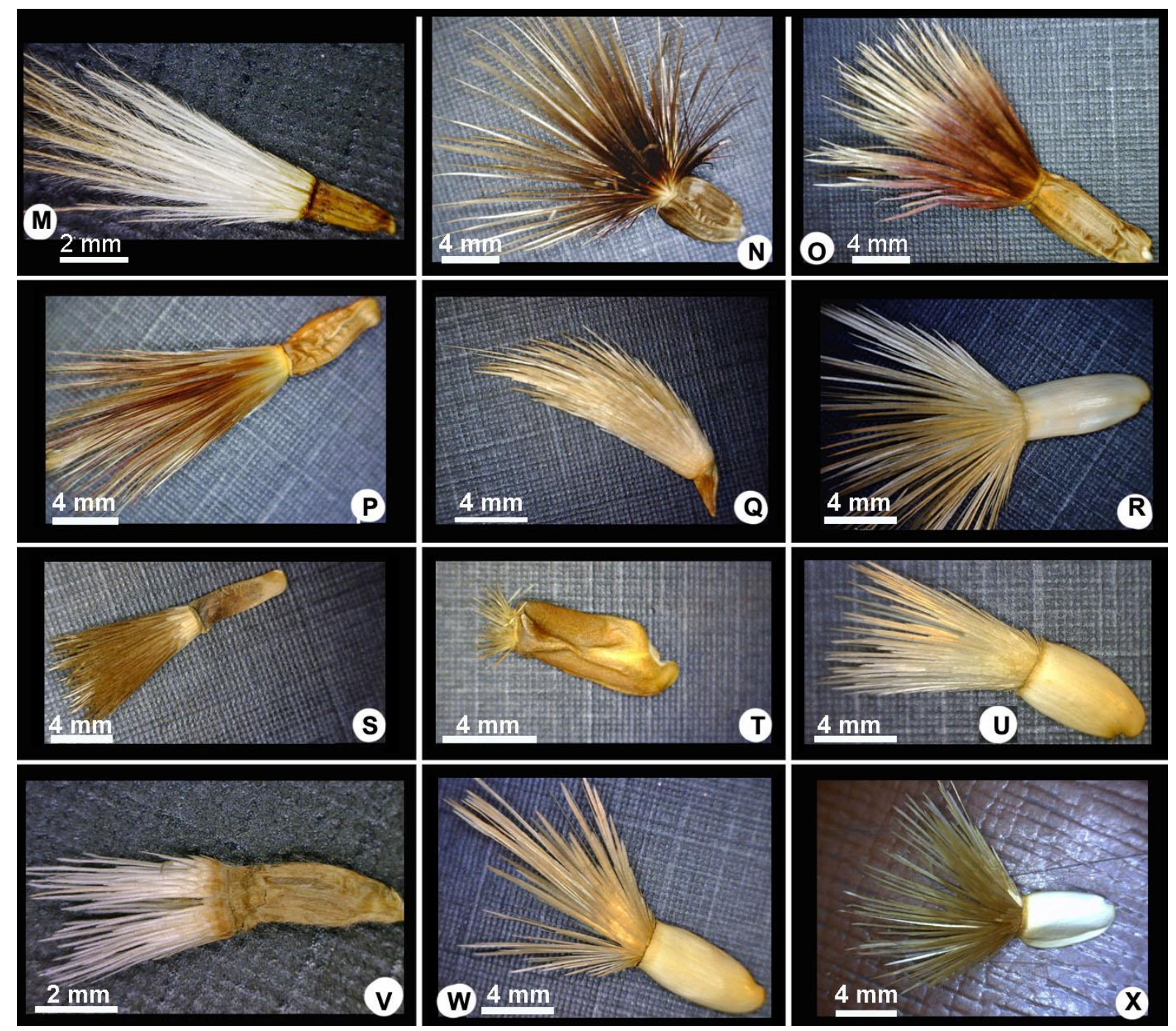

Fig. 3. M. Centaurea glastifolia. N. C. aucheri subsp. aucheri. O. C. aucheri subsp. szowitsii. P. C. aucheri subsp. elburzensis. Q. C. albonitens. R. C. regia. S. C. imperialis. T. C. phlomoides. U. C. gigantea. V. C. geluensis. W. C. amadanensis. X. C. nemeci. 
Table 3. Characteristics used for achene differentiation (measurement in $\mathrm{mm}$ ).

Abbreviations: $\mathbf{P . L}=$ pappus length, $\mathbf{P . C}=$ pappus color, $\mathbf{P . F}=$ pappus form, $\mathbf{P} . \mathbf{T}=$ pappus type, $\mathbf{I} . \mathbf{D} . \mathbf{P}=$ inner dense pappus, $\mathbf{N D}=$ not denticulate, $\mathbf{C o .} \mathbf{L}=$ connection length, $\mathbf{C o .} \mathbf{C}=$ connection color, $\mathbf{C o .} \mathbf{F}=$ connection form, P.L/A.L=pappus length/achene length, $\mathbf{S}=$ scabrous, $\mathbf{D}=$ denticulate, $\mathbf{P}=$ plumose, - = glabrous, +/-=pubsent, +=normal, ++=fairly high, +++=highly.

\begin{tabular}{|c|c|c|c|c|c|c|c|c|c|c|}
\hline Section & species & P.L & P.C & P.F & P.T & ت & $\stackrel{2}{\dot{\theta}}$ & Co.C & $\stackrel{2}{0^{\prime}}$ & $\overrightarrow{0}$ \\
\hline Centaurea & C. lachnopus & 11 & $\begin{array}{l}\text { milky } \\
\text { white }\end{array}$ & straight & $\mathrm{S}$ & - & 2.3 & $\begin{array}{c}\text { dark brown, } \\
\text { margined }\end{array}$ & $\mathrm{D}$ & 1.5 \\
\hline \multirow[t]{2}{*}{ Stizolophus } & $\begin{array}{l}\text { C. balsamita } \\
\text { subsp. } \\
\text { balsamita }\end{array}$ & 4.3 & $\begin{array}{l}\text { brownish } \\
\text { cream }\end{array}$ & straight & $\mathrm{S}$ & - & 1.5 & $\begin{array}{l}\text { light cream, } \\
\text { margined }\end{array}$ & $\mathrm{D}$ & 0.9 \\
\hline & $\begin{array}{l}\text { C. balsamita } \\
\text { subsp. } \\
\text { kermanensis }\end{array}$ & 2.7 & $\begin{array}{l}\text { Brownish } \\
\text { golden }\end{array}$ & straight & $\mathrm{S}$ & - & 1.2 & $\begin{array}{l}\text { light cream, } \\
\text { margined }\end{array}$ & $\mathrm{D}$ & 0.7 \\
\hline \multirow[t]{2}{*}{ Acrolophus } & C. ovina & 0.9 & white & straight & $\mathrm{S}$ & - & 1.12 & $\begin{array}{l}\text { light cream } \\
\text { no margin }\end{array}$ & $\mathrm{D}$ & 0.2 \\
\hline & C.aggregata & 3.1 & white & straight & $\mathrm{S}$ & - & 1.2 & brown margined & $\mathrm{D}$ & 1.1 \\
\hline $\begin{array}{l}\text { Ammoсуапи } \\
s\end{array}$ & $\begin{array}{l}\text { C. } \\
\text { ammocyanus }\end{array}$ & 3.4 & white & straight & $\mathrm{S}$ & - & 1.05 & $\begin{array}{l}\text { golden cream } \\
\text { margined }\end{array}$ & $\mathrm{D}$ & 1.1 \\
\hline Hyalea & C. pulchella & 4.2 & $\begin{array}{c}\text { shining } \\
\text { white }\end{array}$ & straight & $\mathrm{S}$ & + & 1.1 & no margin & $\mathrm{D}$ & 1.9 \\
\hline Jaceae & C. hyrcanica & 1.3 & yellow & $\begin{array}{l}\text { short\& } \\
\text { straight }\end{array}$ & $\mathrm{S}$ & - & 0.9 & no margin & $\mathrm{D}$ & 0.4 \\
\hline Cheirolepis & C. kotschyi & 13.01 & brown & ray form & $\mathrm{P}$ & - & 2.2 & cream margin & $\mathrm{D}$ & 2.1 \\
\hline \multirow{3}{*}{$\begin{array}{l}\text { Rhizocalathi } \\
\text { um }\end{array}$} & C. rhizantha & 1.86 & white & straight & $\mathrm{S}$ & - & 1.3 & no margin & $\mathrm{D}$ & 0.3 \\
\hline & C. ustulata & 2.4 & $\begin{array}{l}\text { honey } \\
\text { brown }\end{array}$ & $\begin{array}{l}\text { short \& } \\
\text { ray form }\end{array}$ & $\mathrm{S}$ & - & 1.6 & no margin & $\mathrm{D}$ & 0.4 \\
\hline & C. ispahanica & 3.9 & $\begin{array}{l}\text { honey } \\
\text { brown }\end{array}$ & $\begin{array}{l}\text { short \& } \\
\text { straight }\end{array}$ & $S$ & - & 2.1 & no margin & $\mathrm{D}$ & 0.4 \\
\hline Chartolepis & C. glastifolia & 9.3 & $\begin{array}{l}\text { cream } \\
\text { white }\end{array}$ & straight & highly $\mathrm{P}$ & - & 0.6 & brown margin & $\mathrm{D}$ & 4.8 \\
\hline \multirow[t]{4}{*}{$\begin{array}{l}\text { Рhаеорарри } \\
s\end{array}$} & $\begin{array}{l}\text { C. aucheri/ } \\
\text { aucheri }\end{array}$ & 12 & $\begin{array}{l}\text { brown } \\
\text { purple }\end{array}$ & $\begin{array}{l}\text { highly } \\
\text { ray form }\end{array}$ & $\mathrm{P}$ & - & 2.3 & cream margin & $\mathrm{D}$ & 2 \\
\hline & $\begin{array}{l}\text { C. aucheri/ } \\
\text { szowitsii }\end{array}$ & 12.02 & $\begin{array}{l}\text { cream, } \\
\text { purple }\end{array}$ & ray form & $\mathrm{P}$ & - & 2.3 & brown margin & $\mathrm{D}$ & 1.6 \\
\hline & $\begin{array}{l}\text { C.aucheri/ } \\
\text { elbursensis }\end{array}$ & 15.4 & $\begin{array}{l}\text { cream } \\
\text { purple }\end{array}$ & $\begin{array}{l}\text { straight } \\
\text { spreadin } \\
\mathrm{g}\end{array}$ & $\mathrm{S}$ & - & 1.8 & $\begin{array}{l}\text { dark brown } \\
\text { margin }\end{array}$ & $\mathrm{D}$ & 2.2 \\
\hline & C. albunitense & 13 & white & $\begin{array}{l}\text { broom } \\
\text { shape }\end{array}$ & $\mathrm{S}$ & - & 1.4 & $\begin{array}{c}\text { white brown } \\
\text { margin }\end{array}$ & $\mathrm{D}$ & 4.5 \\
\hline \multirow[t]{2}{*}{ Cynaroides } & C. regia & 11.5 & cream & ray form & $\mathrm{S}$ & - & 2.9 & no margin & $\mathrm{D}$ & 1.5 \\
\hline & C. imperialis & 9 & $\begin{array}{c}\text { white, } \\
\text { inner }\end{array}$ & straight & $\mathrm{S}$ & - & 1.7 & dark margin & $\mathrm{D}$ & 1.6 \\
\hline \multirow[t]{3}{*}{ Cynaroides } & C. gigantea & 9.2 & white & straight & $\mathrm{S}$ & - & 2.5 & no margin & $\mathrm{D}$ & 1.5 \\
\hline & C. phlomoides & 2.2 & $\begin{array}{l}\text { white } \\
\text { honey }\end{array}$ & $\begin{array}{l}\text { short } \& \\
\text { ray form }\end{array}$ & $\mathrm{S}$ & - & 1.5 & $\begin{array}{c}\text { yellow } \\
\text { margined }\end{array}$ & $\mathrm{D}$ & 0.3 \\
\hline & C. geluensis & 4.2 & $\begin{array}{l}\text { cream } \\
\text { white }\end{array}$ & ray form & $\mathrm{S}$ & - & 1.08 & $\begin{array}{c}\text { red brown } \\
\text { margined }\end{array}$ & $\mathrm{D}$ & 1.02 \\
\hline \multirow[t]{2}{*}{ Paraphysis } & $\begin{array}{l}C . \\
\text { amadanensis }\end{array}$ & 11 & cream & ray form & $\mathrm{S}$ & - & 2.5 & $\begin{array}{c}\text { dark brown } \\
\text { margined }\end{array}$ & $\mathrm{D}$ & 1.5 \\
\hline & C. nemecii & 10.4 & $\begin{array}{l}\text { golden } \\
\text { honey }\end{array}$ & ray form & $\mathrm{S}$ & - & 2.4 & golden margined & $\mathrm{D}$ & 1.8 \\
\hline \multirow[t]{3}{*}{ Microlophus } & C. behen & 6.5 & white & $\begin{array}{c}\text { semi } \\
\text { straight }\end{array}$ & $\mathrm{S}$ & - & 1.6 & no margin & $\mathrm{D}$ & 1.5 \\
\hline & C. pabotii & 5.3 & white & straight & $\mathrm{S}$ & - & 1.6 & no margin & $\mathrm{D}$ & 1.2 \\
\hline & C. koeieana & 5.5 & white & straight & $\mathrm{S}$ & - & 1.5 & brown margined & $\mathrm{D}$ & 2.6 \\
\hline \multirow[t]{2}{*}{ Mesocentron } & C. solstitialis & 4.6 & white & ray form & $\mathrm{S}$ & + & 1.1 & no margin & $\mathrm{D}$ & 1.8 \\
\hline & $\begin{array}{l}\text { C. } \\
\text { pseudosinaica }\end{array}$ & 3.6 & $\begin{array}{l}\text { honey } \\
\text { color }\end{array}$ & $\begin{array}{c}\text { semi ray } \\
\text { form }\end{array}$ & $\mathrm{S}$ & - & 1.2 & no margin & $\mathrm{D}$ & 1.2 \\
\hline \multirow[t]{2}{*}{ Calcitrapa } & C. iberica & 1.16 & white & $\begin{array}{l}\text { short \& } \\
\text { semi ray }\end{array}$ & $\mathrm{S}$ & - & 1.01 & $\begin{array}{c}\text { dark brown } \\
\text { margined }\end{array}$ & $\mathrm{D}$ & 0.3 \\
\hline & C. hyalolepis & 3.2 & white & semi ray & $\mathrm{S}$ & - & 0.9 & yellow margin & $\mathrm{D}$ & 1.3 \\
\hline
\end{tabular}


Table 3. continue ...

\begin{tabular}{|c|c|c|c|c|c|c|c|c|c|c|}
\hline Section & species & P.L & P.C & P.F & P.T & $\ddot{\theta}$ & $\overbrace{0}^{2}$ & Co.C & $\stackrel{2}{0}$ & D \\
\hline Tetramorphaea & C. bruguierana & 2.2 & white & semi ray & $S$ & + & 0.2 & $\begin{array}{l}\text { brown } \\
\text { margin }\end{array}$ & $\mathrm{D}$ & 1.08 \\
\hline \multirow[t]{3}{*}{ Acrocenteron } & C. sosnowskyi & 8 & $\begin{array}{l}\text { honey } \\
\text { cream }\end{array}$ & ray form & $S$ & + & 1.93 & $\begin{array}{c}\text { no } \\
\text { margin }\end{array}$ & $\mathrm{D}$ & 1.48 \\
\hline & $\begin{array}{l}\text { C. } \\
\text { kandavanensis }\end{array}$ & 8.3 & white & $\begin{array}{l}\text { semi ray } \\
\text { form }\end{array}$ & $\mathrm{S}$ & + & 1.81 & $\begin{array}{c}\text { no } \\
\text { margin }\end{array}$ & $\mathrm{D}$ & 1.5 \\
\hline & C. luristanica & 17.8 & $\begin{array}{l}\text { milky } \\
\text { white }\end{array}$ & semi ray & $S$ & + & 2.3 & $\begin{array}{c}\text { no } \\
\text { margin }\end{array}$ & $\mathrm{D}$ & 2.8 \\
\hline \multirow[t]{2}{*}{ Psephelloideae } & C. leuzeoides & 10 & black & ray form & $S$ & + & 2.7 & $\begin{array}{c}\text { no } \\
\text { margin }\end{array}$ & $\mathrm{D}$ & 1.2 \\
\hline & C. gilanica & 7.7 & $\begin{array}{l}\text { cream } \\
\text { purple }\end{array}$ & $\begin{array}{l}\text { semi ray } \\
\text { form }\end{array}$ & $\mathrm{S}$ & - & 2.1 & $\begin{array}{l}\text { brown } \\
\text { margin }\end{array}$ & ND & 1.1 \\
\hline Psephellus & C. zuvandica & 0.5 & yellow & $\begin{array}{l}\text { too short } \\
\& \text { ray } \\
\text { form }\end{array}$ & $S$ & - & 1.6 & $\begin{array}{l}\text { brown } \\
\text { margin }\end{array}$ & $\mathrm{D}$ & 0.1 \\
\hline Amblyopogon & C. incanescens & 1.7 & white & $\begin{array}{l}\text { dense \& } \\
\text { diagonal }\end{array}$ & $S$ & - & 2.2 & $\begin{array}{l}\text { black, } \\
\text { brown } \\
\text { margin }\end{array}$ & $\mathrm{D}$ & 0.2 \\
\hline Odontolophoideae & $\begin{array}{l}\text { C. } \\
\text { phaeopappoides }\end{array}$ & 2.2 & $\begin{array}{l}\text { brown } \\
\text { honey }\end{array}$ & ray form & $S$ & + & 1.6 & $\begin{array}{l}\text { honey } \\
\text { margin }\end{array}$ & $\mathrm{D}$ & 0.5 \\
\hline Uralepis & C. gaubea & 7.4 & white & $\begin{array}{l}\text { semi ray } \\
\text { form }\end{array}$ & $S$ & - & 1.6 & $\begin{array}{l}\text { red } \\
\text { brown } \\
\text { margin }\end{array}$ & $\mathrm{D}$ & 2.6 \\
\hline Xanthopsis & $\begin{array}{l}C . \\
\text { xanthocephala }\end{array}$ & 2.2 & purple & $\begin{array}{l}\text { short\& } \\
\text { semi ray } \\
\text { form }\end{array}$ & $S$ & + & 1.3 & $\begin{array}{l}\text { black } \\
\text { margin }\end{array}$ & $\mathrm{D}$ & 0.3 \\
\hline Cyanus & C. cyanus & 6.4 & white & ray form & $S$ & + & 2.1 & $\begin{array}{l}\text { cream } \\
\text { margin }\end{array}$ & $\mathrm{D}$ & 1.3 \\
\hline \multirow[t]{4}{*}{ Cyanus } & $\begin{array}{l}\text { C. } \\
\text { cheiranthifolia }\end{array}$ & 1.3 & $\begin{array}{l}\text { amber } \\
\text { colored }\end{array}$ & $\begin{array}{l}\text { short \& } \\
\text { semi ray } \\
\text { form }\end{array}$ & $S$ & + & 1.7 & $\begin{array}{c}\text { red } \\
\text { brown } \\
\text { margin }\end{array}$ & $\mathrm{D}$ & 0.3 \\
\hline & C. depressa & 6.2 & white & $\begin{array}{l}\text { semi ray } \\
\text { form }\end{array}$ & $S$ & + & 2.1 & $\begin{array}{l}\text { cream } \\
\text { margin }\end{array}$ & $\mathrm{D}$ & 1.3 \\
\hline & C. elbursensis & 18 & $\begin{array}{l}\text { cream } \\
\text { white }\end{array}$ & $\begin{array}{l}\text { semi ray } \\
\text { form }\end{array}$ & $S$ & - & 2.2 & $\begin{array}{c}\text { dark } \\
\text { brown } \\
\text { margin }\end{array}$ & $\mathrm{D}$ & 3.2 \\
\hline & C. triumphetti & 1.5 & $\begin{array}{l}\text { amber } \\
\text { colored }\end{array}$ & $\begin{array}{l}\text { short \& } \\
\text { straight }\end{array}$ & $S$ & + & 1.6 & $\begin{array}{l}\text { light } \\
\text { margin }\end{array}$ & $\mathrm{D}$ & 0.3 \\
\hline
\end{tabular}

\section{DISSCUSION}

The character states used for achene characterization in different species of the genus Centaurea are thoroughly investigated, and some of them were found to be major key features. Character states derived from the presence of hair on the achene, the presence of appendage, the length and color of the appendage, the length of hilum, the length of achene and its color, the length of pappus, especially in comparison with the length of achene, the presence of inner dense pappus as well as the color and form of the connection area of hilum were found to be important.

Due to the Flora Iranica (Wagenitz, 1980), the identification key generated for the separation of the groups (A-I) were based on characters such as the form and color of appendage and bracts, the number of cilia, the form of leaves, the presence or absence of terminal spine and the duration of the plant. In this paper, the identification key for 24 sections of the genus Centaurea is constructed on the basis of the achene specifications. Three groups are considerably distinct and recognized. Group A is distinguished by having the achene with large and highly observable prominent appendage coming out of hilum. Group B is specified by having the achene with appendage enclosed by the hilum. Moreover, Group $\mathrm{C}$ is specified by the absence of appendage in achene's hilum.

Group A: Achene with large and highly prominent appendage out of hilum

1. Hilum appendage red..............sect. Stizolophus

- Hilum appendage white.........................2

2. Achene with margin in connection to pappus ....3

- Achene with no margin in connection to pappus.5

3. Achene hairy on its surface.....................4

- Achene glabrous....................sect. Centaurea 
4. Inner pappus dense and scale like. sect. Odontoh

- Inner pappus loose sect.

5. Pappus shorter than the achene sect Rhizocalathium

- Pappus longer than the achene sect. Mesocentron $=C$. pseudosinaica

Group B: Achene with small appendage, but not prominent in coming out of hilum

1. Achene with margin in connection to pappus.....2

- Achene without margin in connection to pappus...

.......................sect. Acrolophus $=$ C. ovina

2. Pappus length / achene length more than $1 \ldots \ldots 3$

- Pappus length / achene length less than 1 ......... 4

3. Hilum truncate........................sect. Uralepis

- Hilum round.... .5

4. Achene denticulate in pappus connection area....6

- Achene not as above......sect. Cyanus $=$ C. cyanus

5. Hilum scabrous................................. 7

- Hilum smooth ................................... 8

6. Pappus with inner dense row.................... .sect. Cynaroides $=C$. depressa

- Pappus without inner dense row. ..sect. Cyanus $=$ C. elbursensis

7. Pappus with inner dense row...... sect. Xanthopsis

- Pappus without inner dense.

...................sect. Cynaroids $=$ C. phlomoides

8. Achene hairy..................................10

- Achene glabrous................................9

9. Pappus purple...................sect. Phaeopappus

- Pappus not purple. sect. Paraphysis $=C$. amadanensis

10. Pappus purple..sect. Psephelloideae $=C$. gilanica

- Pappus not purple....................................11

11. Achene shiny white...sect. Paraphysis $=C$. nemecii

- Achene Brown .12

12. Pappus length more than $5 \mathrm{~mm}$. sect. Cynaroides $=C$. imperialis

- Pappus length less than $5 \mathrm{~mm}$.

13. pappus with inner dense row. ...sect. Cyanus $=$ C. . cheiranthifolia

- Pappus with no inner dense.

...sect. Ammocyanus, sect. Acrolophus=C. aggregate

Group C: Achene without appendage

1. Pappus with inner dense row.....................2

- Pappus with no inner dense row..................5

2. Achene scarcely hairy..................sect. Hyalea

- Achene glabrous....................................3

3. Achene margined on pappus connection area...........................sect. Tetramorphaea

- Achene no margined........................... 4

4. Achene white, pappus black. .sect. Psephelloideae $=$ C. leuzeoides
- Achene cream-colored, pappus white.

....................sect. Mesocentron $=C$. solstitialis

5. Achene hairy ................................6

- Achene glabrous.........sect. Cynaroides $=$ C. regia

6. Hilum hairy..........................sect. Calcitrapa

- Hium glabrous .................................... 7

7. Achene margined on pappus connection area.....9

- Achene no margined............................... 8

8. Hilum concave................................ 10

- Hilum angled......................sect. Microlophus

9. Achene-pappus small opening..........sect. Jaceae

- Achene-pappus large opening..................... sect. Cynaroides $=C$. gigantean

10. Pappus longer than the achene ..............11

- Pappus shorter than the achene or the same.....12

11. Achene hairy.....................sect. Chartolepis

- Achene glabrous...................sect. Cheirolepis

12. Pappus truncate-oblique..........sect. Amblyopogon

- Pappus not as above.

.sect. Cynaroides $=C$. geluensis

According to Tables 2 and 3, some differences are observed in some species among sections. In sect. Acrolophus, in C. ovina (Fig. 2 E) no margin is seen in achene pappus connection area, while $C$. aggregata (Fig. 2 D) has a brown margin. Moreover, based on Tables 2 and 3, in C. ovina, pappus length is shorter than the achene length, while in C. aggregata pappus is longer. Therefore, the ratio of pappus length to achene length is more than 1. One important point to mention is that the similarity between sects. Acrolophus and Ammocyanus, based on the features studied in Flora Iranica (Wagenitz 1980), is mirrored in the high similarity between $C$. ammocyanus (Fig. $2 \mathrm{~F}$ ) and $C$. aggregata in the section mentioned, in the features studied here (Tables $2 \& 3$ ). In sect. Phaeopappus, despite the categorization of $C$. albonitens with $C$. aucheri in the mentioned section, white broom form pappus is seen (Fig. 3 Q), though the other subspecies are interesting and recognized by having ray form purple pappus (Fig. $3 \mathrm{~N}, \mathrm{O} \& \mathrm{P}$ ). Moreover, pappus in C. albonitens is much longer in comparison with achene length.

Sect. Cynaroides is one of the most varied sections, as many different character states were observed among its five species. C. regia is the only species among all others with no hairs on its achene surface (Fig. 3 Q), while the others have hairs as $C$. imperialis (Fig. $3 \mathrm{R}$ ) and C. gigantea (Fig. $3 \mathrm{~T}$ ) are specified by having scarce hairs and $C$. phlomoides (Fig. $3 \mathrm{R}$ ) and C. geluensis (Fig. 3, V) are fully covered with hairs on their achene surface.

In sect. Cynaroides, the species C. phlomoides has distinct morphological differences in the characters studied compared with other species of the section. 
In addition, different bracts and capitulum size are additional morphological traits to convince us to separate $C$. phlomoides from the other members of this section and treat it as a new section.

Three species namely $C$. regia, $C$. geluensis and $C$. gigantea have no appendage, while the other species do. Moreover, $C$. regia and $C$. gigantea have no margin. In all species in sect. Cynaroides, pappus is obviously longer than the achene, however, pappus length in $C$. phlomoides is much shorter than the achene length. Furthermore, according to Tables 2 and 3, hilum length in $C$. phlomoides is much longer than the others. The ratio of pappus length to achene length is less than one in the mentioned species, while in others it is more than 1.
In sect. Paraphysis, C. amadanensis has no hairs on the achene (Fig. $3 \mathrm{~W}$ ), and the color of the achene is creamy, but in C. nemecii achene is hairy and white (Fig. $3 \mathrm{X}$ ). These two species are morphologically close to each other, however, they are separated on the basis of the presence of pedicel in $C$. amadanensis or the lack of pedicel in C. nemesi.

In sect. Microlophus, achenes in species $C$. behen, C. pabotii and C. koeieana (Fig. 4 A, B and C) were thoroughly investigated. In all these three species, the surface of achene is hairy, but achene in $C$. koeieana is overwhelmingly hairy. Also, in the two species mentioned first, the form of hilum is corner angled, while hilum in $C$. koeieana is scanned. Moreover, the length of

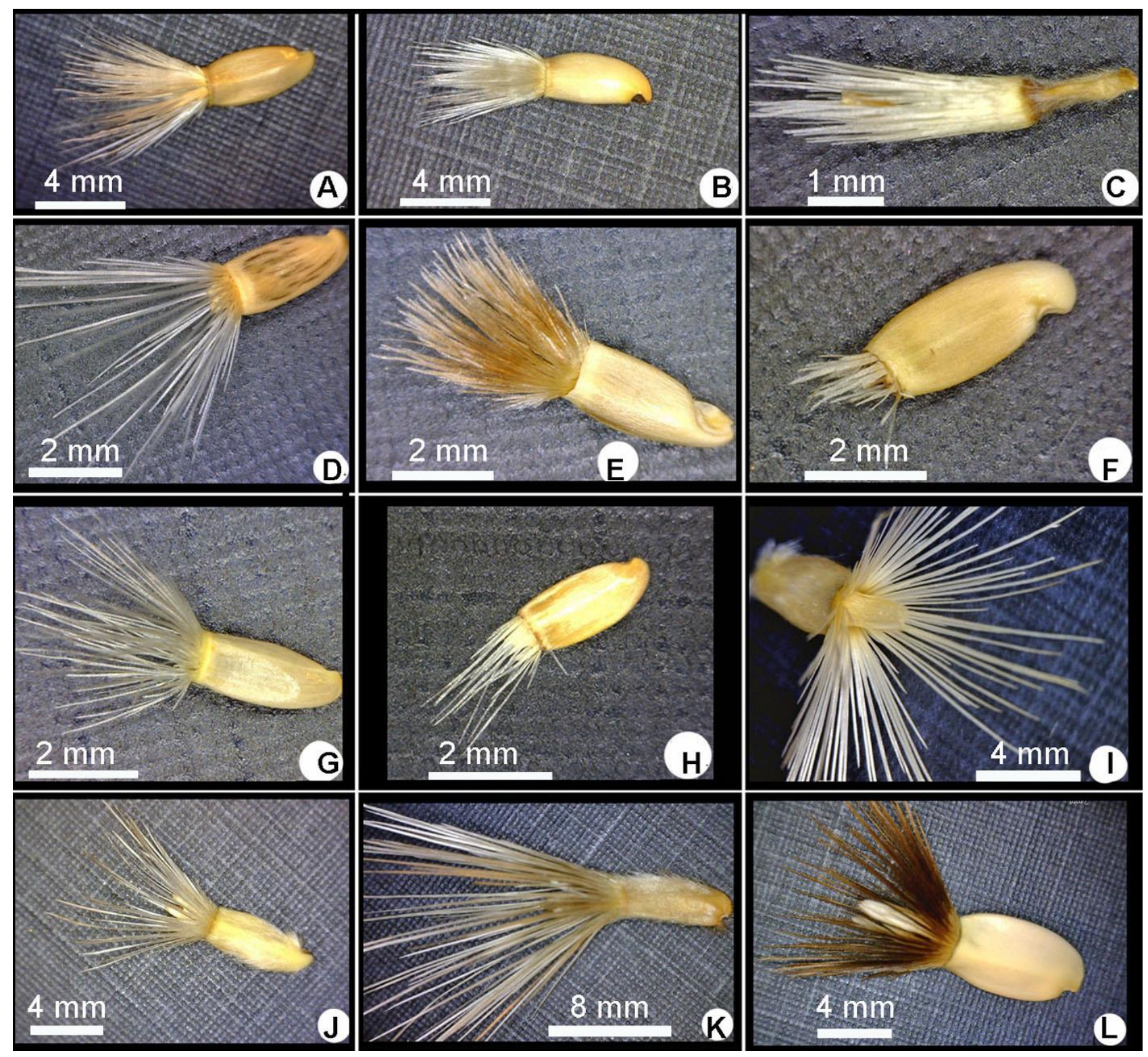

Fig. 4. A. Centaurea behen. B. C. Pabotii. C. C. Koeiana. D. C. solstitialis subsp. solstitialis. E. C. pseudosinaica. F. C. iberica. G. C. hyalolepis. H. C. bruguierana. I. C. sosnowskyi. J. C. kandavanensis. K. C. luristanica. L. C. leuzeoides. 


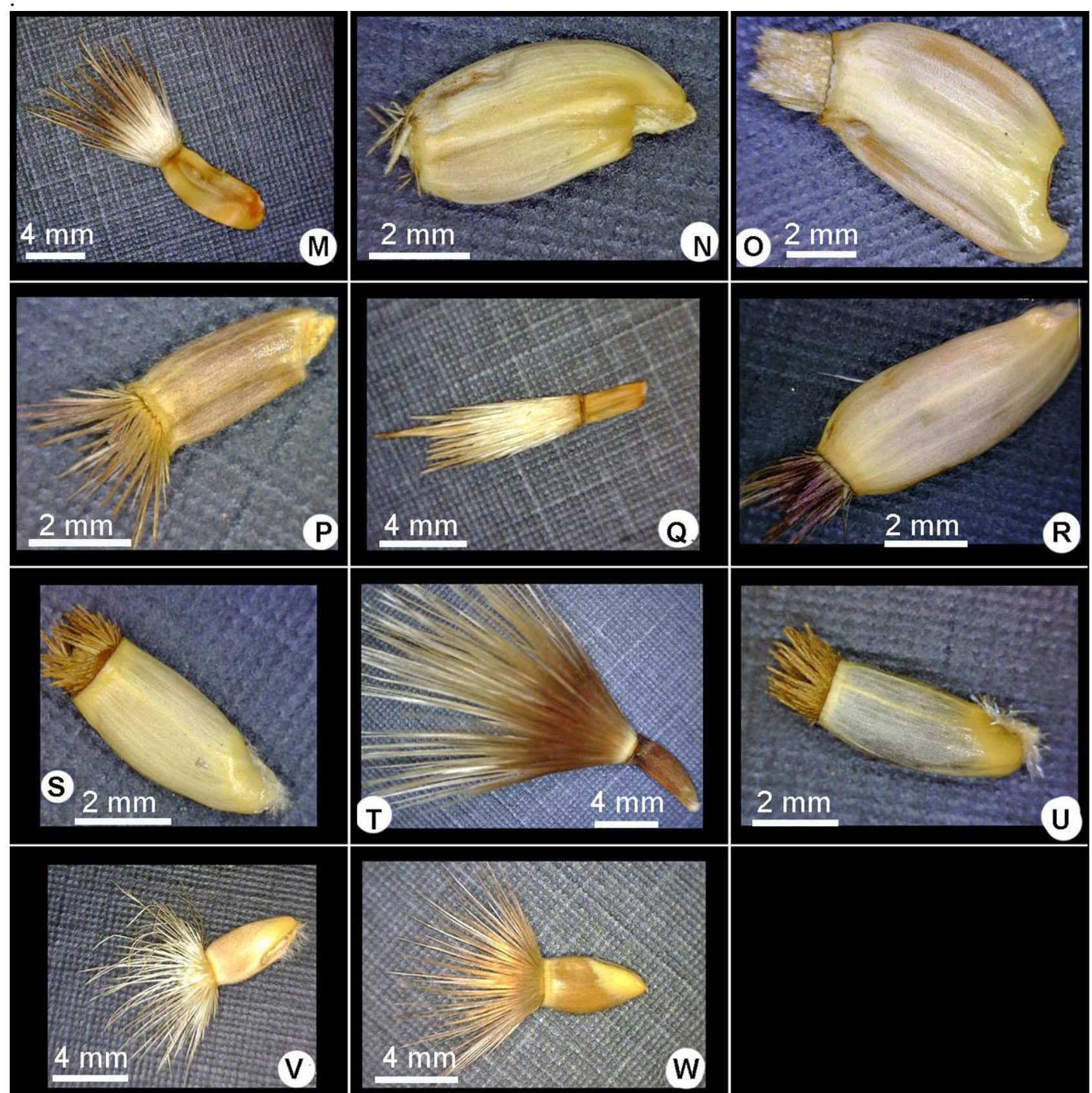

Fig. 5. M. Centaurea gilanica. N. C. zuvandica. O. C. incanescens. P. C. phaeopappoides. Q. C. gaubae. R. C. xantocephala. S. C. cheiranthifolia. T. C. elburzensis. U. C. triumfettii. V. C. depressa. W. C. cyanus.

pappus is almost 3 times more than achene length. This section is homogenous in the characters studied. The appendage of bracts is without hilum and all flowers are yellow. Their achenes are also very similar.

In sect. Mesocentron, an interesting reversed relation is seen in the presence of inner dense pappus and appendage. It means that, in $C$. solstitialis (Fig. 4 D), no appendage is seen but the inner dense pappus is obvious with white achene, while $C$. pseudosinaica (Fig. $4 \mathrm{E}$ ) is seen with prominent appendage, no inner dense pappus and brown achene. These two species are similar in that they have yellow flowers and 'bract appendage' that leads to long acute spines, but different because of their geographical distribution, as the first one is an Irano-Turanian element while the second one is a Sahara-Sindian element. Their achenes are completely different.

In sect. Calcitrapa, two species namely $C$. iberica (Fig. $4 \mathrm{~F}$ ) and $C$. hyalolepis (Fig. $4 \mathrm{G}$ ) are thoroughly investigated. The most important criteria to be used in the comparison between these two is the length of pappus. This means that, in the first species, the length of pappus is shorter than that of the achene, while in $C$. hyalolepis the pappus is longer. Therefore, the ratio is more than 1 in the former, but less than 1 in the latter. In addition, short 
amount of hairs can be seen in hilum area of $C$. iberica.

Sect. Psephelloideae, is a group with a lot of differences among its species. C. leuzeoides (Fig. 4 1): 1-lacks hairs on achene, 2-lacks appendage, 3shiny white achene, 4- has black pappus, 5-lacks margin in pappus-achene connection area, 6- has denticulate pappus-achene connection form,7- has inner dense pappus

C. gilanica (Fig. $5 \mathrm{M}$ ): 1. has hairs on achene surface, 2. has appendage, 3. the achene color is yellow brown, 4. has cream-colored purple pappus, 5 . has brown margin in pappus-achene connection area, 6. Achene-pappus connection is not denticulate, 7. lacks inner dense pappus.

Sect. Amblyopogon with only one species, i.e. C. incanescens, have the largest hilum as compared with all other sections, short, dense and in diagonal form pappus (Fig. 5 O).

Sect. Xanthopsis, besides phaeopappus, is famous for its purple pappus.

The other interesting section is cyanus: the species thoroughly observed included $C$. cheiranthifoli, $C$. depressa, $C$. cyanus, $C$. elbursensis and $C$. triumfettii. The prominent differences investigated were addressed as follows.

In C. cheiranthifolia (Fig. 5 S) no hairs were seen on achene, while the others have hairs on their achene.

Hilum length in C. cyanus (Fig. $5 \mathrm{~W}$ ) is the biggest in comparison with other species in this section. The achene shape in C. cyanus (Fig. $5 \mathrm{~W}$ ) is triangular while others have oblong shape. C. elbursensis (Fig. $5 \mathrm{~T}$ ) is the only species in this group without inner dense central pappus, while others have the feature. Pappus in C. cheiranthifolia (Fig. $5 \mathrm{~S}$ ) and $C$. triumfetti (Fig. $5 \mathrm{U}$ ) are shorter than the achene, while in other species of the mentioned section, pappus are long enough to consider. Interestingly, in C. elbursensis, pappus is much longer in comparison with achene length.

Based on the discussion, despite the fact that each section contains a lot of species with many similarities in achene's morphology, many differences in species in many of those sections have been observed.

Based on the studied morphological traits, especially the morphology of achene, these traits are useful for the delimitation of the species. Although a new key has been provided for categorizing the sections, achene morphological traits were found to be inadequate. In each section type, achenes are different among some species even when similarities were observed in other morphological traits.

Morphologically, some traits such as the presence or absence of appendage of bracts, central bract appendage form, the number of cilias around appendage, the color of cilias and the color of flowers can be useful for the delimitation of sections. In addition, achene characteristics can be useful for the delimitation of the species in the genus Centaurea.

\section{ACKNOWLEDGEMENT}

We thank anonymous reviewers for their useful comments.

\section{REFERENCES}

Boissier, E. 1875. Centaurea L. - In: Flora Orientalis 3: 614-696. - H. Georg, Geneva \& Basileae.

Bona, M. 2014. Achene characteristics of Turkish Centaurea (Asteraceae) and their systematic application. - Bangladesh J. Bot. 43: 163-168.

Bremer, B., Bremer, K., Chase, M., Fay, M., Reveal, J., Soltis D. and Stevens, P. 2009. An update of the Angiosperm Phylogeny Group classification for the orders and families of flowering plants: APG III. Bot. J. Linn. Soc. 161: 105-121.

Davis, P.H., Mill, R. and Tan, K. (eds.). 1988. Flora of Turkey and the East Aegean Islands, (Supplement) 10: 489-501. - Edinburgh University Press, Edinburgh.

Ghahreman, A. and Attar, F. 1999. Biodiversity of plant species in Iran. - Tehran University Publications, Tehran.

Heywood, V.H. 1979. Flowering plants of the world. Oxford University Press, Oxford.

Hickey, M. and King, C.J. 1981. 100 families of flowering plants. - Cambridge University Press, Cambridge.

Judd, W.S., Campbell, C.S., Kellogg, E.A., Stevens, P.F. and M.J. Donoghue. 2008. Plant systematics: A phylogenetic approach (Third Edition). Sinauer Associates, Inc., Sunderland, Massachusetts.

Maleev P. V. 1971. Centaurea L. In: Komarov V.L (ed.), Flora of U.S.S.R 28: 368-577. - Academy of Science of the U.S.S.R, Moscow \& Leningrad.

Negaresh, K. and Rahiminejad, M.R. 2014. A contribution to the taxonomy of Centaurea sect. Cynaroides (Asteraceae, Cardueae-Centaureinae) in Iran. - Phytotaxa 158: 229-244.

Ranjbar, M. and Negaresh, K. 2014. A revision of Centaurea sect. Centaurea (Asteraceae) from Iran. Turk. J. Bot. 38: 969-987.

Shabestari, E.S.B., Attar, F., Riahi, H., and Sheidai, M. 2013a. Pollen morphology of Centaurea L. (Asteraceae) in Iran. - Acta Bot. Brasil. 27: 669-679.

Shabestari, E.S.B., Attar, F., Riahi, H. and Sheidai, M. 2013b. Seed morphology of the Centaurea species (Asteraceae) in Iran. - Phytol. Balcan 19: 209-214.

Wagenitz, G. 1986. Centaurea in South-West Asia: patterns of distribution and diversity, Proceedings of the Royal Society of Edinburgh. Section B. Biological Sciences 89: 11-21. 
Wagenitz, G. 1980. Centaurea L. - In: Rechinger, K.H. (ed.) Flora Iranica. 139b: 356-362. - Akademische Druck-und Verlagsanstalt, Graz.
Wagenitz, G. 1975. Centaurea L. - In: Davis, P.H. (ed.) Flora of Turkey and the East Aegean Islands Vol. 5: 465-586. - Edinburgh University Press, Edinburgh.

How to cite this article:

Rakizadeh, S., Attar, F. and Sotoodeh, A. 2019. Taxonomic significance of achene morphology on the Centaurea L. (Asteraceae). - Nova Biol. Reperta 6: 352-366. 